\title{
Design of inductors with high inductance values for resonant piezoelectric damping
}

\author{
B. Lossouarn ${ }^{\mathrm{a}, *}$, M. Aucejo ${ }^{\mathrm{a}}$, J.-F. Deü ${ }^{\mathrm{a}}$, B. Multon ${ }^{\mathrm{b}}$ \\ ${ }^{a}$ Structural Mechanics and Coupled Systems Laboratory, Cnam \\ 292 Rue Saint-Martin, 75003 Paris, France \\ ${ }^{b}$ SATIE, École normale supérieure de Rennes, UBL, CNRS \\ Campus de Ker Lann, 35170 Bruz, France
}

\begin{abstract}
The resonant piezoelectric shunt requires specific inductance and resistance values in order to reach an optimum in terms of vibration reduction. Yet, practical limits appear in the low frequency range: the required inductance and the corresponding quality factor are often too high to be satisfied with standard passive components. In this paper, inductors are designed with closed magnetic cores made of high permeability materials. Those components are successively integrated into a piezoelectric shunt dedicated to vibration control of a cantilever beam. It is shown that custom designs can definitely extend the application of passive resonant shunt strategies to lower frequency.
\end{abstract}

Keywords: Inductor design, Resonant shunt, Piezoelectricity, Passive damping, Magnetic cores

\section{Nomenclature}

$\hat{L}_{s}(\omega) \quad$ Equivalent series inductance

$R(\omega)$ Equivalent series resistance

$\mu$ Permeability

$\mu_{0} \quad$ Vacuum permeability

$\mu_{\mathrm{e}} \quad$ Relative effective permeability

$\omega$ Angular frequency

$\omega_{\mathrm{e}} \quad$ Resonant shunt angular frequency

$\omega_{\mathrm{O}} \quad$ Natural angular frequency in open circuit

$\omega_{\mathrm{S}} \quad$ Natural angular frequency in short circuit

$\rho$ Resistivity of the conductor

$\xi \quad$ Magnetic flux

$\xi_{\mathrm{e}} \quad$ Effective cross-sectional area

$A_{\mathrm{w}} \quad$ Window area

$A_{L} \quad$ Permeance

$B^{L} \quad$ Magnetic flux density

$B_{\text {sat }} \quad$ Saturation flux density

$C^{\text {sat }} \quad$ Blocked piezoelectric capacitance

$c_{Q} \quad$ Criterion for the choice of the core

$D$ Mechanical displacement transfer function

$d_{\mathrm{w}} \quad$ Wire diameter

$e \quad$ Global piezoelectric coefficient

$E_{\max }$ Maximum energy in the inductor

$F$ Force applied to the structure

$f_{\mathrm{O}} \quad$ Natural frequency in open circuit

$G \quad$ Charge displacement transfer function

$H \quad$ Magnetic field strength

$i_{B} \quad$ Electrical current

$I_{\mathrm{ngax}}^{B} \quad$ Maximum current before saturation

$I_{\max }^{\text {max }}$ Maximum current before overheating

*Corresponding author

Email address: boris.lossouarn@cnam.fr (B. Lossouarn) 


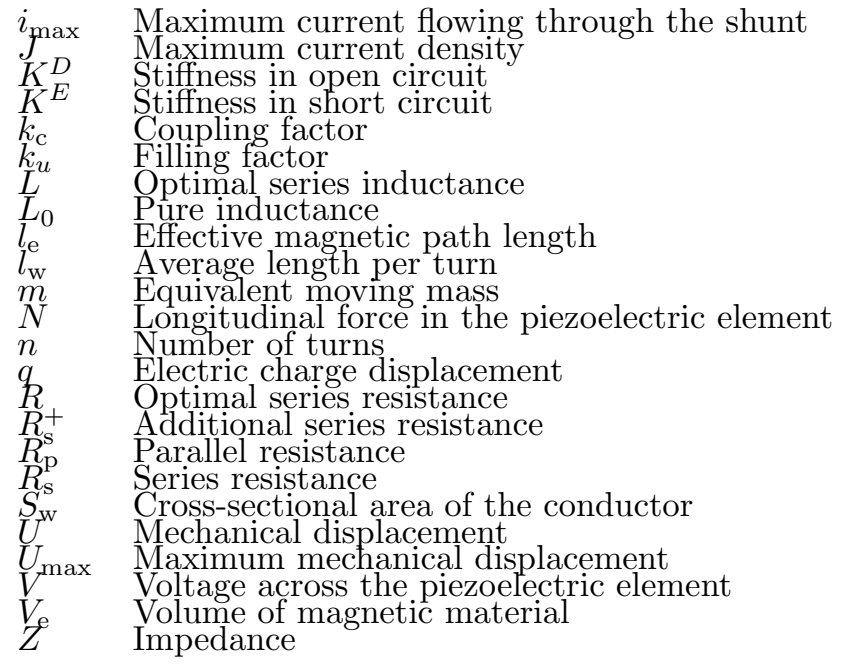

\section{Introduction}

Structural vibration damping occurs when shunting piezoelectric material with passive electrical circuits. This can be realized with the resistive and resonant shunts described by Hagood and von Flotow [1. It essentially consists in converting the mechanical energy of a vibrating structure into electrical energy, which is then dissipated into a resistor. In addition, the resonant shunt requires an inductor that is combined to the piezoelectric capacitance in order to generate an electrical resonance. Similarly to what is observed with a tuned mass damper [2, 3], the electrical resonance enhances the energy transfer when it is tuned to the mechanical mode to control. Consequently, a passive and potentially lightweight solution can provide significant vibration reduction without strong modification of the mechanical structure. This control strategy was first applied through single-mode shunts [1, 4, 8] and then extended to multi-mode shunts [9].

A drawback of the resonant shunt technique is that practical applications generally require large inductance values. The notion of large inductance is not clearly defined in the shunt damping literature because, depending on the authors, it can be in a range of $0.1 \mathrm{H}, 10 \mathrm{H}$ or even $1000 \mathrm{H} \mathrm{[6,} \mathrm{15,} \mathrm{16.} \mathrm{In} \mathrm{any} \mathrm{case,}$ the standard inductor series are usually limited to $0.5 \mathrm{H}$, which cannot satisfy most of the resonant shunt applications. Fleming et al. [16] proposed to use additional capacitance across the electrodes of the piezoelectric patches in order to decrease the required inductance. However, they also show that this solution induces a reduction of the damping performance. Furthermore, standard inductors present another limit: they offer an internal resistance which is usually too large for resonant shunt applications [17]. The challenging inductance and resistance requirements explain why most of the experimental validations involving resonant shunts are performed with synthetic inductors [6 9, 15, 17, 20] or with a current source [11]13]. The use of those active circuits pushes back the limits of physical inductors but it questions the practical implementation of a purely passive resonant shunt damping.

A passive inductor is commonly made of a coil of conductive wire wound around a magnetic circuit. Contrary to what has been sometimes stated [8, 16, passive inductors above $1 \mathrm{H}$ are easily feasible with closed magnetic cores [21, 22. Moreover, the direct equivalence between large inductance and large weight or volume [7, 17, 19, 20, is unfunded because the selection of a magnetic core depends on the energy that has to be stored into the component. For vibration control applications involving relatively low energy transfers, high permeability ferrite cores are available and give access to large inductance values [22, 23]. Furthermore, numerous magnetic materials with larger permeabilities are also available. For example, some nanocrystalline alloys exhibit permeabilities up to ten times the highest ferrite permeabilities. The required inductance can then be obtained with a fewer number of turns, which reduces the internal resistance of the component. The use of closed magnetic circuits with high permeability materials thus enables the design of inductors with large inductance values and high quality factors [23]. This allows the application of piezoelectric damping strategies with purely passive components [24, 25]. 


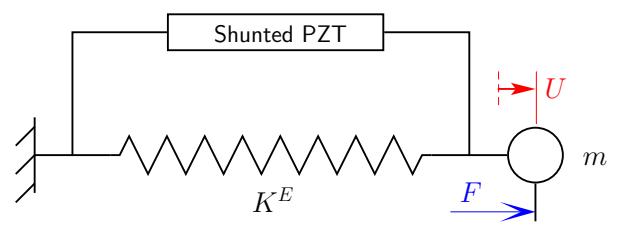

Figure 1: Spring-mass model illustrating a control with shunted piezoelectric material.

The main objective of this paper is to show that resonant piezoelectric damping can be implemented with passive inductors. First, we recall the main results related the resonant shunts. An optimal tuning of the electrical component is defined by minimizing the displacement of the structure to control. This also leads to novel results concerning the quantification of the energy that has to be stored into the inductor. Yet, we note that practical applications usually require very high inductance values that cannot be satisfied with standard inductors. The next sections then shows that closed magnetic cores can be of great interest for resonant piezoelectric damping. The main characteristics of passive inductors are described and it is explained how to select a suitable magnetic core from resonant shunt specifications. The last section of the paper is then devoted to the design of inductors for the control of a cantilever beam with a pair of piezoelectric patches. A ferrite core and a nanocrystalline toroid are selected and used to damp the first two bending modes of the considered beam.

\section{Resonant shunt damping}

Considering resonant piezoelectric shunts, optimal inductance and resistance values are found from a minimization of a transfer function involving the mechanical displacement to control. The maximum current flowing through the shunt is also quantified for later design of the electrical components. Yet, an example experimental setup shows that the required inductance values cannot be satisfied with standard passive components.

\subsection{Spring-mass model}

The resonant piezoelectric shunt is first illustrated through the control of a unimodal structure represented by a spring-mass system. As shown in Fig. 1, a shunted piezoelectric element is connected in parallel with a spring of stiffness $K^{E}$, representing the stiffness of the whole structure when the piezoelectric transducer is short-circuited. The piezoelectric coupling is defined from

$$
\begin{aligned}
& N=K^{E} U-e V \\
& q=e U+C^{\varepsilon} V
\end{aligned},
$$

where $N$ is the longitudinal force in the piezoelectric material, $U$ is the displacement of the mass $m, V$ is the voltage between its electrodes and $q$ represents the electric charge displacement. The constant $e$ which is expressed in $\mathrm{N} / \mathrm{V}$ or in $\mathrm{C} / \mathrm{m}$ is the global piezoelectric coefficient and $C^{\varepsilon}$ is the blocked capacitance, that is the piezoelectric capacitance measured when preventing displacement of the mechanical system $(U=0)$. If $F$ is an external force applied to the mass $m$, it is found from Fig. 1 that $m \ddot{U}=F-N$ and Eq. (1) gives

$$
\begin{aligned}
& m \ddot{U}=F-K^{E} U+e V \\
& q=e U+C^{\varepsilon} V
\end{aligned}
$$

One can then define a natural angular frequency in short circuit: $\omega_{\mathrm{S}}=\sqrt{K^{E} / m}$. This refers to the resonance of the mechanical structure when the piezoelectric element is short-circuited $(V=0)$. If the shunt is made of an inductor $L$ in series with a resistor $R$, the impedance $Z(\omega)=R+\mathrm{j} \omega L$ leads to

$$
V=-R \dot{q}-L \ddot{q} .
$$




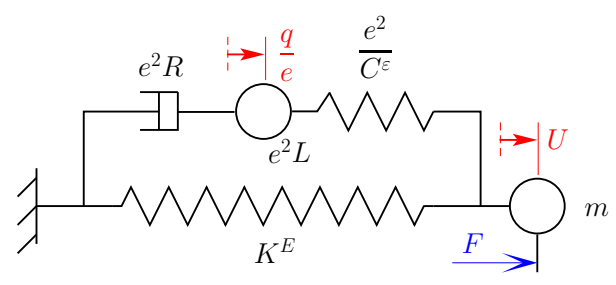

(a)

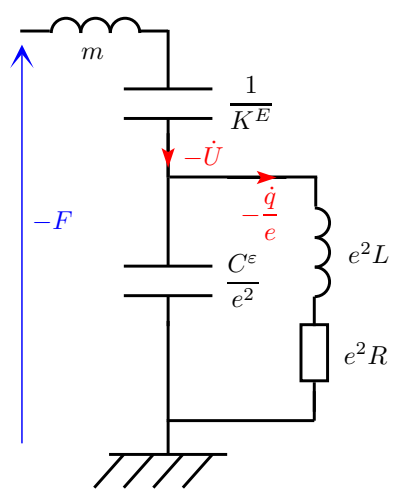

(b)

Figure 2: Analogous models representing a resonant piezoelectric shunt: (a) Mechanical representation. (b) Electrical representation.

In the present study, we consider that the inductor is not subjected to magnetic saturation, so that the inductance $L$ does not depend on the current amplitude. Substituting Eq. (3) into Eq. (2) gives

$$
\begin{aligned}
& m \ddot{U}=F-K^{E} U-\frac{e^{2}}{C^{\varepsilon}}\left(U-\frac{q}{e}\right) \\
& e^{2} L \frac{\ddot{q}}{e}=-e^{2} R \frac{\dot{q}}{e}+\frac{e^{2}}{C^{\varepsilon}}\left(U-\frac{q}{e}\right)
\end{aligned},
$$

which can be illustrated by the mechanical model in Fig. 2(a). It is found a model that directly shows the analogy of the resonant shunt with a tuned mass damper [2, 3. Remark that the damper is not between the two moving mass but between the added mass and the ground, meaning that an infinite damping is equivalent to an open-circuit case.

The mechanical models in Fig. 22(a) can be converted into its electrical analogues by reformulating Eq. (4) as

$$
\begin{aligned}
& -F=-\mathrm{j} \omega m \dot{U}-\frac{K^{E}}{\mathrm{j} \omega} \dot{U}+\frac{e^{2}}{\mathrm{j} \omega C^{\varepsilon}}\left(\frac{\dot{q}}{e}-\dot{U}\right) \\
& -\mathrm{j} \omega e^{2} L \frac{\dot{q}}{e}-e^{2} R \frac{\dot{q}}{e}=\frac{e^{2}}{\mathrm{j} \omega C^{\varepsilon}}\left(\frac{\dot{q}}{e}-\dot{U}\right)
\end{aligned}
$$

in order to introduce the electrical circuit in Fig. 2(b). Equation (5) shows that $-\dot{q} / e$ corresponds to the current flowing through the resulting RL branch and the inductor $m$ represents the mass of the mechanical structure. When comparing Figs. 2(a) and 2(b), there are equivalences between mass and inductor, damper and resistor and spring and capacitor. This refers to the direct electromechanical analogy [26- 28].

\subsection{Optimization of the resonant shunt}

When coupling a resonant piezoelectric shunt to a mechanical structure, the damping performance can be optimized by choosing suitable resistance and inductance values. If we focus on a min-max optimization on the displacement $U$ of the mass $m$, we want to minimize the maximum of the displacement transfer function $U / F$, where the input excitation is a harmonic force $F$ of constant amplitude. Several methods were proposed to define optimal values for $L$ and $R$ [1, 6, 29]. It is remarked that they all give relatively close results when dealing with moderate values of the coupling factor $k_{\mathrm{c}}=\sqrt{e^{2} /\left(K^{E} C^{\varepsilon}\right)}$ 6, 29. Here, the method proposed by Thomas et al. [6] is considered. Equation (4) is first written as

$$
\begin{aligned}
& m \ddot{U}+K^{D} U=F+\frac{e}{C^{\varepsilon}} q \\
& L \ddot{q}+R \dot{q}+\frac{1}{C^{\varepsilon}} q=\frac{e}{C^{\varepsilon}} U
\end{aligned},
$$


where $K^{D}=K^{E}+e^{2} / C^{\varepsilon}$. The constant $K^{D}$ is the stiffness of the structure in Fig. 1 when the piezoelectric element is open-circuited, i.e. $q=0$. Then, Eq. (6) is equivalent to

$$
\begin{aligned}
& \frac{1}{\omega_{\mathrm{O}}^{2}} \ddot{U}+U=\frac{F}{K^{D}}+\frac{e}{K^{D} C^{\varepsilon}} q \\
& \frac{1}{\omega_{\mathrm{e}}^{2}} \ddot{q}+\frac{2 \xi_{\mathrm{e}}}{\omega_{\mathrm{e}}} \dot{q}+q=e U
\end{aligned},
$$

where $\omega_{\mathrm{e}}=\frac{1}{\sqrt{L C^{\varepsilon}}}$ is the resonant shunt angular frequency, $\xi_{\mathrm{e}}=\frac{R}{2} \sqrt{\frac{C^{\varepsilon}}{L}}$ is the damping factor and $\omega_{\mathrm{O}}=\sqrt{K^{D} / m}$ is the natural angular frequency in open-circuit. Recall that $\omega_{\mathrm{S}}=\sqrt{K^{E} / m}$, the coupling factor is thus defined from the open- and short-circuit natural frequencies as

$$
k_{\mathrm{c}}=\sqrt{\frac{e^{2}}{K^{E} C^{\varepsilon}}}=\sqrt{\frac{K^{D}-K^{E}}{K^{E}}}=\sqrt{\frac{\omega_{\mathrm{O}}^{2}-\omega_{S}^{2}}{\omega_{S}^{2}}} .
$$

Under harmonic excitation, one obtains from Eq. (7) that

$$
\left(1-\frac{\omega^{2}}{\omega_{\mathrm{O}}^{2}}\right) U-\frac{e}{K^{D} C^{\varepsilon}} q=\frac{F}{K^{D}} \quad \text { and } \quad q=\frac{e}{1-\frac{\omega^{2}}{\omega_{\mathrm{e}}^{2}}+2 \mathrm{j} \xi_{\mathrm{e}} \frac{\omega}{\omega_{\mathrm{e}}}} U .
$$

By remarking from Eq. (8) that $e^{2} /\left(K^{D} C^{\varepsilon}\right)=\left(\omega_{\mathrm{O}}^{2}-\omega_{S}^{2}\right) / \omega_{\mathrm{O}}^{2}$, the dimensionless displacement transfer function is expressed as

$$
D(\omega)=\frac{U}{F / K^{D}}=\frac{1-\frac{\omega^{2}}{\omega_{\mathrm{e}}^{2}}+2 \mathrm{j} \xi_{\mathrm{e}} \frac{\omega}{\omega_{\mathrm{e}}}}{\frac{\omega_{\mathrm{S}}^{2}}{\omega_{\mathrm{O}}^{2}}-\left(\frac{1}{\omega_{\mathrm{O}}^{2}}+\frac{1}{\omega_{\mathrm{e}}^{2}}\right) \omega^{2}+\frac{\omega^{4}}{\omega_{\mathrm{O}}^{2} \omega_{\mathrm{e}}^{2}}+2 \mathrm{j} \xi_{\mathrm{e}} \frac{\omega}{\omega_{\mathrm{e}}}\left(1-\frac{\omega^{2}}{\omega_{\mathrm{O}}^{2}}\right)},
$$

which gives

$$
|D(\omega)|^{2}=\frac{\left[1-\frac{\omega^{2}}{\omega_{\mathrm{e}}^{2}}\right]^{2}+4 \frac{\omega^{2}}{\omega_{\mathrm{e}}^{2}} \xi_{\mathrm{e}}^{2}}{\left[\frac{\omega_{\mathrm{S}}^{2}}{\omega_{\mathrm{O}}^{2}}-\left(\frac{1}{\omega_{\mathrm{O}}^{2}}+\frac{1}{\omega_{\mathrm{e}}^{2}}\right) \omega^{2}+\frac{\omega^{4}}{\omega_{\mathrm{O}}^{2} \omega_{\mathrm{e}}^{2}}\right]^{2}+4 \frac{\omega^{2}}{\omega_{\mathrm{e}}^{2}}\left[1-\frac{\omega^{2}}{\omega_{\mathrm{O}}^{2}}\right]^{2} \xi_{\mathrm{e}}^{2}} .
$$

The tuning of the resonant shunt consists in determining the values of $\omega_{\mathrm{e}}$ and $\xi_{\mathrm{e}}$ that minimize the maximum of $|D(\omega)|$. Analytic calculations presented in [6] give

$$
\omega_{\mathrm{e}}=\omega_{\mathrm{O}} \quad \text { and } \quad \xi_{\mathrm{e}}=\sqrt{\frac{3}{8}} k_{\mathrm{c}}
$$

In the end, Eq. (12) leads to the optimal inductance and resistance:

$$
L=\frac{1}{C^{\varepsilon} \omega_{\mathrm{O}}^{2}} \quad \text { and } \quad R=\sqrt{\frac{3}{2}} \frac{k_{\mathrm{c}}}{C^{\varepsilon} \omega_{\mathrm{O}}},
$$

where $k_{\mathrm{c}}$ is the coupling factor that has been defined in Eq. (8).

The interest of the resonant piezoelectric control is illustrated by observing the modulus of the transfer function $D(\omega)$ around $\omega_{\mathrm{O}}$, the natural frequency in open circuit. First, Fig. 3 shows that the use of a resonant shunt without any resistance creates an antiresonance at $\omega_{\mathrm{e}}$, which is equal to $\omega_{\mathrm{O}}$ when the inductance is tuned to its optimal value. Yet, the resonant shunt adds a new degree of freedom, as seen in the equivalent mechanical model in Fig. 2(a). This explains why two resonances appear on both sides of the antiresonance. The displacement is thus reduced around $\omega_{\mathrm{O}}$ but it is significantly increased around the new resonances. Finally, the resistance given in Eq. 130 is added into the shunt to get a vibration reduction over a broader frequency range. 


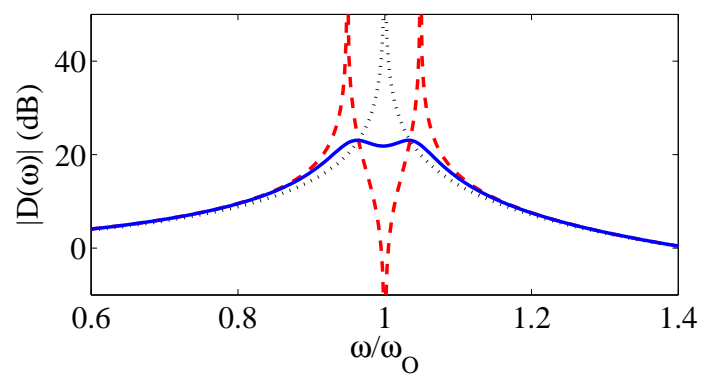

Figure 3: Modulus of the transfer function $D(\omega):(\ldots)$ with an open circuit, (- -) with the optimal inductance and no resistance, $(-)$ with the optimal inductance and the optimal resistance.

\subsection{Current flowing through the inductor}

Once optimal values of the electrical components are known, it is important to quantify the current flowing through the resonant shunt in order to choose a suitable inductor. Equation (9) shows that the electric charge displacement $q$ is related to the mechanical displacement $U$ through the dimensionless transfer function

$$
G(\omega)=\frac{q / e}{U}=\frac{1}{1-\frac{\omega^{2}}{\omega_{\mathrm{e}}^{2}}+2 \mathrm{j} \xi_{\mathrm{e}} \frac{\omega}{\omega_{\mathrm{e}}}},
$$

whose square modulus is

$$
|G(\omega)|^{2}=\frac{1}{\left[1-\frac{\omega^{2}}{\omega_{\mathrm{e}}^{2}}\right]^{2}+4 \frac{\omega^{2}}{\omega_{\mathrm{e}}^{2}} \xi_{\mathrm{e}}^{2}} .
$$

The maximum of $|G(\omega)|$ is reached when $\omega=\omega_{\mathrm{e}} \sqrt{1-2 \xi_{\mathrm{e}}^{2}} \approx \omega_{\mathrm{e}}$. Consequently, $\xi_{\mathrm{e}}=\sqrt{3 / 8} k_{\mathrm{c}}$ gives

$$
|G(\omega)|_{\max } \approx \sqrt{\frac{2}{3}} \frac{1}{k_{\mathrm{c}}}
$$

In the end, with $i_{\max } \approx\left|i\left(\omega_{\mathrm{O}}\right)\right|=\omega_{\mathrm{O}}\left|q\left(\omega_{\mathrm{O}}\right)\right|$, the maximum current flowing through the shunt is obtained from Eqs. (14) and (16) as

$$
i_{\max }=\sqrt{\frac{2}{3}} \frac{\omega_{\mathrm{O}}}{k_{\mathrm{c}}}|e| U_{\max }
$$

The mechanical displacement $U$ has been chosen as a reference to illustrate typical applications where the displacement has to be maintained below a maximum value $U_{\max }$. This value is generally known because it is defined by the designer of the system. Furthermore, Eq. (2) shows that the global coupling coefficient is $e=-C^{\varepsilon} V / U$ in open circuit. If $e$ has not been calculated from the characteristics of the considered system, it can still be obtained experimentally from the ratio between the voltage and the displacement in open circuit.

The maximum current given in Eq. (17) allows quantifying the maximum energy that has to be stored into the inductor

$$
E_{\max }=\frac{1}{2} L i_{\max }^{2}
$$

From Eqs. (8), (13), (17) and (18), it is remarked that the maximum energy can also be expressed as

$$
E_{\max }=\frac{1}{3} K^{E} U_{\max }^{2}
$$

In the end, Eq. (19) shows that the magnetic component has to be able to store two thirds of the total mechanical energy. Note that this result is based on the maximum displacement $U_{\max }$ observed once the 


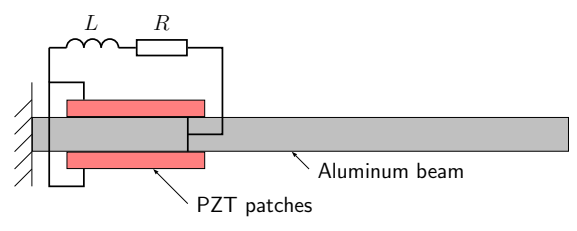

(a)

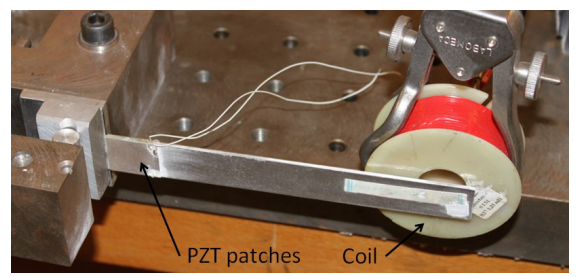

(b)

Figure 4: Experimental setup: (a) Cantilever beam with two collocated piezoelectric patches connected to a resonant shunt. (b) Picture of the actual setup showing the coil excitation system.

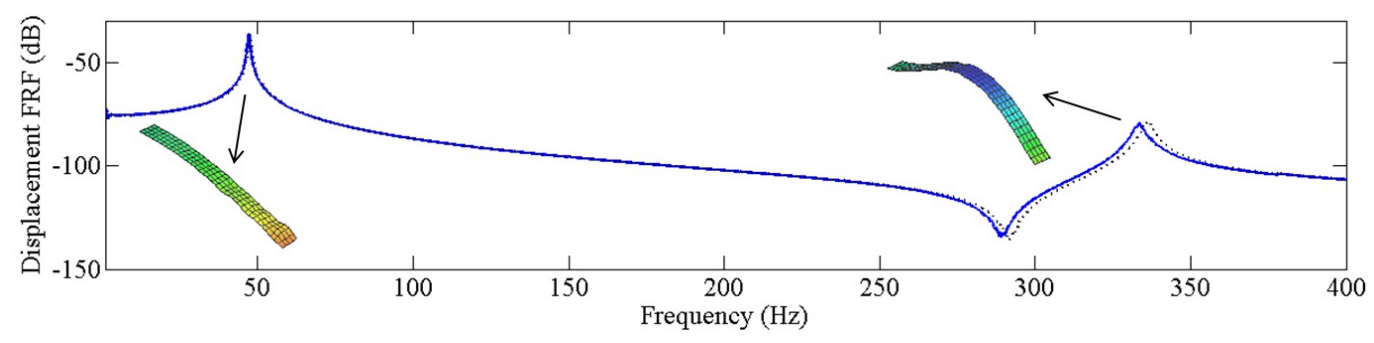

Figure 5: Displacement FRFs and corresponding operating deflection shapes obtained experimentally: ( - ) with short-circuited patches, $(\cdots)$ with open-circuited patches.

structure is controlled with a resonant shunt satisfying the tuning condition in Eq. (13). The resistance value has a direct influence on the energy transfer: reducing the resistance increases the current flowing through the shunt, which increases the amount of energy that has to be stored into the inductor.

\subsection{Experimental investigations}

An example setup involving a cantilever beam controlled by a pair of PZT patches is shown in Fig. 4(a). The beam is excited by a non-contact system involving a coil and a magnet at the free end of the beam. This setup was presented by Thomas et al. [6] for experiments involving resistive and resonant piezoelectric shunts. The aluminum beam is $170 \mathrm{~mm}$ long, $25 \mathrm{~mm}$ wide and $2 \mathrm{~mm}$ thick. The two PZT patches are made of PIC 151 material, they are $25 \mathrm{~mm}$ long, $20 \mathrm{~mm}$ wide and $0.5 \mathrm{~mm}$ thick. As seen in Fig. 4(b), the collocated patches are placed near the clamping area for maximizing their effect [6, 30]. In the present setup, they are polarized in identical directions and connected in parallel for the control of bending modes [1, 31. The velocity of the beam is measured with a laser vibrometer. The frequency response functions are then obtained by referring to the current in the coil which is considered proportional to the input force over the frequency range of interest [6]. A scanning process allows the experimental characterization of the operating deflection shapes. Two bending modes are identified below $400 \mathrm{~Hz}$. They are represented in Fig. 5 with the frequency response function (FRF) related to the displacement at the free end of the beam.

The blocked capacitance is approximated by direct measurement of the piezoelectric capacitance at $100 \mathrm{~Hz}$ and $20^{\circ} \mathrm{C}$ when no bending motion is allowed and it is found $C^{\varepsilon}=34.4 \mathrm{nF}$. From the FRFs in Fig. 5 and from Eqs. (8) and (13), it becomes possible to extract optimal inductance and resistance for the control of the first two bending modes. The results are given in Table 1 , which presents for each mode the resonance frequencies in short and open circuit, $f_{\mathrm{S}}$ and $f_{\mathrm{O}}$, the coupling factor $k_{\mathrm{c}}$ and the resulting series inductance $L$ and series resistance $R$. We remark that the required inductance exceeds easily $0.5 \mathrm{H}$, which can be considered as an upper limit for standard inductors provided by electronic component distributors. High inductance could be eventually satisfied with inductors in series but this would require numerous components that would largely exceed the optimal resistance 32. This highlights a practical limit of the resonant shunt damping strategy when considering low frequency applications. Yet, it is shown in the following section 
Table 1: Natural frequencies, piezoelectric coupling factors and optimal shunt specifications for the first two bending modes.

\begin{tabular}{|c|c|c|c|c|c|}
\cline { 2 - 6 } \multicolumn{1}{c|}{} & $\begin{array}{c}\boldsymbol{f}_{\mathrm{S}} \\
(\mathrm{Hz})\end{array}$ & $\begin{array}{c}\boldsymbol{f}_{\mathrm{O}} \\
(\mathrm{Hz})\end{array}$ & $\begin{array}{c}\boldsymbol{k}_{\mathrm{c}} \\
(-)\end{array}$ & $\begin{array}{c}\boldsymbol{L} \\
(\mathrm{H})\end{array}$ & $\begin{array}{c}\boldsymbol{R} \\
(\mathrm{k} \Omega)\end{array}$ \\
\hline Mode 1 & 47.07 & 47.46 & 0.129 & 327 & 15.4 \\
\hline Mode 2 & 333.6 & 336.1 & 0.123 & 6.52 & 2.07 \\
\hline
\end{tabular}

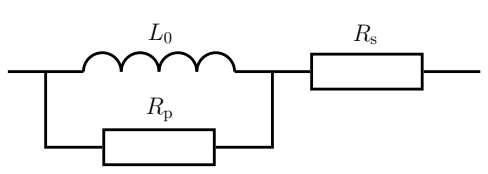

(a)

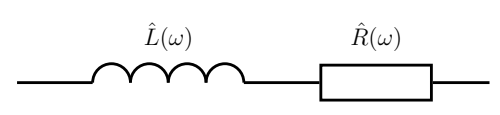

(b)

Figure 6: Electrical circuit models of an inductor: (a) Model with a series resistance and a parallel resistance. (b) Model involving an equivalent series inductance and an equivalent series resistance.

that for standard vibration amplitudes which involves relatively low energy transfers, suitable inductors of reasonable size can be designed.

\section{Design of passive inductors}

An electrical model for an inductor with both series and parallel resistance is given. The basics of inductor design are then presented with the classical equations governing magnetic circuits. The inductance is defined from the properties of the magnetic core, which is selected in order to satisfy the electrical specifications of the resonant shunt.

\subsection{Equivalent circuit models}

A suitable model for a passive inductors is obtained by considering a pure inductance $L_{0}$ combined with a resistance $R_{\mathrm{s}}$ in series and a resistance $R_{\mathrm{p}}$ in parallel, as represented in Fig. 6(a). The resistance $R_{\mathrm{s}}$ approximates the low frequency copper losses, i.e. the Joule heating in the wire that forms the inductor. The parallel resistance $R_{\mathrm{p}}$ represents the magnetic losses. Those are due to iron losses in the magnetic material (hysteretic losses and the eddy current losses) as well as losses in the winding through skin and proximity effects [21, 22. The equivalent series resistance $\hat{R}(\omega)$ is represented in Fig. 6(b) together with $\hat{L}(\omega)$, which is the equivalent series inductance. Both values come from the impedance of the electrical model in Fig. 6(a), which is recast in $Z(\omega)=\hat{R}(\omega)+j \omega \hat{L}(\omega)$ where

$$
\begin{aligned}
& \hat{L}(\omega)=\frac{L_{0}}{1+\left(\frac{L_{0} \omega}{R_{\mathrm{p}}}\right)^{2}} \\
& \hat{R}(\omega)=R_{\mathrm{s}}+\frac{R_{\mathrm{p}}}{1+\left(\frac{R_{\mathrm{p}}}{L_{0} \omega}\right)^{2}} .
\end{aligned}
$$

From this formulation, it is remarked that the equivalent series inductance and resistance necessarily depend on frequency. This effect is due to the magnetic losses that have been represented by the parallel resistance $R_{\mathrm{p}}$. If $R_{\mathrm{p}}$ is constant, $\hat{R}(\omega)$ increases and $\hat{L}(\omega)$ decreases when $\omega$ increases. This explains the frequency dependence observed when measuring the equivalent inductance and resistance of passive inductors involving non-negligible magnetic losses. 


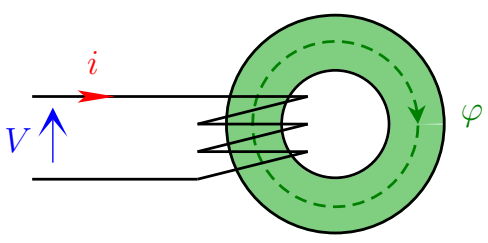

(a)

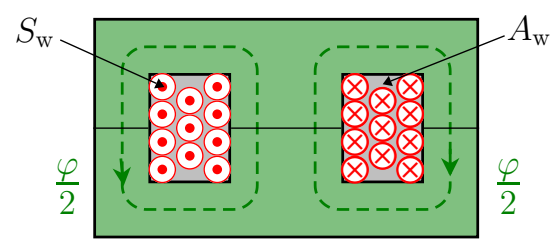

(b)

Figure 7: Two inductor designs: (a) Toroidal core. (b) RM core.

\subsection{Basics of electromagnetism}

When considering a magnetic core without air gap and made of a linear and homogeneous magnetic material of permeability $\mu$, the equations related to the magnetic flux density $B$, the magnetic field strength $H$ and the magnetic flux $\varphi$ can be written as follows:

$$
B=\mu H, \quad \varphi=B A_{\mathrm{e}} \quad \text { and } \quad H l_{\mathrm{e}}=n i .
$$

The constant $A_{\mathrm{e}}$ defines the effective cross-sectional area of the magnetic core and $l_{\mathrm{e}}$ is the effective magnetic path length. The magnetic flux $\varphi$ is caused by the current $i$ flowing through a conductor, which is wound around the core with $n$ turns. This is represented in Fig. 7(a) in a case involving a toroidal core. With other core geometries, the flux may be split in two magnetic paths, as shown in Fig. 7(b) where a RM core is considered [21, 22]. In any case, a changing magnetic flux is generated by a voltage $V$, which is equal to the time derivative of the total flux going through the $n$ turns of the coil: $V=n \frac{d \varphi}{d t}$. Moreover, the inductance $L$ of an electrical circuit is defined as the ratio of the voltage to the time derivative of the current, i.e. $V=L \frac{d i}{d t}$. As a consequence, the inductance can be expressed from Eq. 21 by

$$
L=A_{L} n^{2}
$$

where $A_{L}=\mu_{\mathrm{e}} \mu_{0} A_{\mathrm{e}} / l_{\mathrm{e}}$ is the permeance. The permeability $\mu$ is defined as the product of the vacuum permeability $\mu_{0}$ times the relative effective permeability $\mu_{\mathrm{e}}$, which is usually given by the core manufacturers together with the constants $A_{\mathrm{e}}$ and $l_{\mathrm{e}}$.

\subsection{Magnetic core selection}

In the previous section, it has been shown that piezoelectric damping applications involving resonant shunts require specific inductance and resistance values. Considering a magnetic core of permeance $A_{L}$, Eq. 22 gives the number of turns that is required to satisfy the inductance $L$. The number of turns is naturally restricted by the fixed window area $A_{\mathrm{w}}$. This limit is quantified by the filling factor

$$
k_{u}=\frac{n S_{\mathrm{w}}}{A_{\mathrm{w}}}
$$

where $S_{\mathrm{w}}$ is the cross-sectional area of the conductor. Even with a full window area, the filling factor $k_{u}$ does not usually exceed 0.5 , especially with very thin wires. This is partly due to the presence of insulation layers that limit the proportion of conductor in the total wire cross-section [21, 22. Another reason is that a wire with circular cross-section cannot entirely fill the available window area. The manufacturing process is also crucial because it determines the winding arrangements. For instance, a handmade coil with disorganized layers leads to a significant reduction of $k_{u}$.

Once the number of turns is defined, a full window area minimizes the resistance of the wire, which is obtained from

$$
R_{\mathrm{s}}=\rho \frac{n l_{\mathrm{w}}}{S_{\mathrm{w}}}=\frac{\rho l_{\mathrm{w}}}{k_{u} A_{\mathrm{w}}} n^{2},
$$


where $l_{\mathrm{w}}$ is the average length per turn and $\rho$ is the resistivity. This shows that the series resistance is minimized by maximizing the filing factor. Yet, as $k_{u}$ is bounded, an inadequate selection of the magnetic core can lead to a case where $R_{\mathrm{s}}$ exceeds the optimal shunt resistance $R$. Furthermore, the copper losses only represent a portion of the total losses in the component [21 23 . Indeed, the influence of the parallel resistance $R_{\mathrm{p}}$ in Fig. 6(a) is usually non-negligible for piezoelectric shunt applications involving high permeability cores 24. We thus need to ensure

$$
\hat{R}(\omega) \leq R
$$

and the shunt can then be tuned by adding an external resistor $R_{\mathrm{s}}^{+}$. The parallel resistance $R_{\mathrm{p}}$ may be difficult to evaluate as it depends on the core material, on the frequency and on the flux density. In the following experiments, its contribution to the equivalent series resistance is clearly below the optimal shunt resistance.

The introduction of a criterion for the choice of a core that is able to satisfy both specifications on $L$ and $R$ is essential. The condition in Eq. (25) necessarily induces that $R_{\mathrm{s}} \leq R$. Then, Eqs. $(22),(23)$ and (24) leads to the definition of a criterion

$$
c_{Q}=\left(\frac{k_{u} A_{L} A_{\mathrm{w}}}{\rho l_{\mathrm{w}}}\right) \frac{R}{L} \geq 1
$$

The criterion $c_{Q}$ is thus calculated from the core specifications and from the electrical properties of the resonant shunt. The filling factor can be set to $k_{u}=0.5$ and $\rho$ to $1.7 \times 10^{-8} \Omega \cdot \mathrm{m}$, which is the resistivity of copper [21]. If $c_{Q}$ is more than one, we can expect a resonant shunt with a sufficiently high quality factor if the contribution of the parallel resistance $R_{\mathrm{p}}$ is not significant. If $c_{Q}$ is below one, the considered core cannot satisfy both $L$ and $R$ requirements. Then, another core with a larger ratio $A_{L} A_{\mathrm{w}} / l_{\mathrm{w}}$ would have to be found.

\subsection{Maximum current and stored energy}

Two practical limits appear when considering the use of passive inductors. First, the magnetic flux density has to remain below the saturation flux density $B_{\text {sat }}$ in order to avoid a reduction of the equivalent inductance value. From Eqs. 21] and 22, this means that

$$
i_{\max }<I_{\max }^{B}=\left(\frac{B_{\mathrm{sat}} A_{\mathrm{e}}}{\sqrt{A_{L}}}\right) \frac{1}{\sqrt{L}},
$$

where $I_{\max }^{B}$ represents the maximum current before saturation. The second limit concerns the root mean square current density $i_{\max } /\left(\sqrt{2} S_{\mathrm{w}}\right)$, which cannot exceed $J$, the maximum current density that would damage the component. Consequently, Eqs. (22) and (23) gives

$$
i_{\max }<I_{\max }^{J}=\left(\sqrt{2} k_{u} J A_{\mathrm{w}} \sqrt{A_{L}}\right) \frac{1}{\sqrt{L}},
$$

where $I_{\max }^{J}$ is the amplitude of the sinusoidal current leading to a root mean square current density equal to $J$. The currents $I_{\max }^{B}$ and $I_{\max }^{J}$ thus need to be considered as upper limits when introducing the chosen inductor into a specific electrical circuit. For practical cases involving high permeance magnetic cores as in the following experiments, it is remarked that $I_{\max }^{B} \ll I_{\max }^{J}$. Magnetic saturation is thus the main limitation that has to be considered when designing the inductors. Note from Eq. (27) that the maximum current $I_{\max }^{B}$ decreases when $A_{L}$ increases. That means that even if high permeance can be used to reach high inductance values, one has to keep in mind that this restrains the maximum energy that can be stored into the component. Indeed, when $i_{\max }=I_{\max }^{B}$, Eqs. 18, 22 and 27) lead to

$$
E_{\max }=\frac{1}{2} L I_{\max }^{B}{ }^{2}=\frac{1}{2} \frac{B_{\mathrm{sat}}^{2}}{\mu_{\mathrm{e}} \mu_{0}} V_{\mathrm{e}},
$$

where $V_{\mathrm{e}}=A_{\mathrm{e}} l_{\mathrm{e}}$ is the volume of magnetic material. If we want to increase the storage capability of the magnetic core, we thus have either to increase its volume or to decrease its effective permeability, provided that Eq. (26) is still satisfied. 


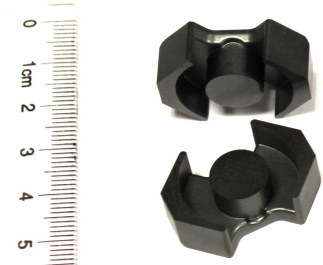

(a)

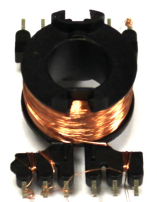

(b)

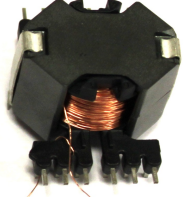

(c)

Figure 8: RM core: (a) Two half parts of the magnetic core. (b) Coil former. (c) Resulting component

Table 2: Geometry and properties of the two magnetic cores.

\begin{tabular}{|c|c|c|c|c|c|c|c|c|}
\cline { 2 - 8 } \multicolumn{1}{c|}{} & $\begin{array}{c}\boldsymbol{A}_{\mathbf{e}} \\
\left(\mathrm{mm}^{2}\right)\end{array}$ & $\begin{array}{c}\boldsymbol{l}_{\mathrm{e}} \\
(\mathrm{mm})\end{array}$ & $\begin{array}{c}\boldsymbol{A}_{\mathrm{w}} \\
\left(\mathrm{mm}^{2}\right)\end{array}$ & $\begin{array}{c}\boldsymbol{l}_{\mathrm{w}} \\
(\mathrm{mm})\end{array}$ & $\begin{array}{c}\boldsymbol{B}_{\mathrm{sat}} \\
(\mathrm{T})\end{array}$ & $\begin{array}{c}\boldsymbol{\mu}_{\mathbf{e}} \\
(-)\end{array}$ & $\begin{array}{c}\boldsymbol{A}_{\boldsymbol{L}} \\
(\mu \mathrm{H})\end{array}$ & $\begin{array}{c}\boldsymbol{E}_{\max } \\
(\mu \mathrm{J})\end{array}$ \\
\hline $\begin{array}{c}\text { RM 10 core } \\
(\mathrm{T} 38)\end{array}$ & 98 & 44 & 41 & 52 & 0.43 & 5720 & 16 & 55 \\
\hline $\begin{array}{c}\mathbf{3 0} \times \mathbf{2 0} \times \mathbf{1 0} \text { toroid } \\
(\text { Vitroperm 500F) }\end{array}$ & 40 & 79 & 236 & 50 & 1.20 & 87500 & 56 & 21 \\
\hline
\end{tabular}

\section{Experimental results}

Magnetic components are selected in order to damp vibration of the considered cantilever beam with a piezoelectric resonant shunt. Suitable inductors involving two different types of closed cores are designed accordingly and connected to the pair of piezoelectric patches.

\subsection{Magnetic cores for the experiments}

Two closed magnetic cores with a similar mass around 22 grams but different shapes and materials are selected. The first core is a RM 10 core made of T38 material (EPCOS). The core is represented in Fig. 8(a) and its geometry is given in Table 2. As seen in Figs. 8(b) and 8(c), a cylindrical coil former facilitates winding and assembly of the magnetic component. As various materials are available for this range of components, the ferrite core providing the larger permeance was selected in order to maximize the criterion $c_{Q}$ given in Eq. 26). This justifies the use of the RM 10 core in T38 ferrite, whose material properties and resulting permeance are given in Table 2. The limit in terms of energy storage is then obtained from Eq. (29). Note that no energy density optimization is given in the present study whose main objective is to show that high inductance values can be reached with passive components. The dimensions of the magnetic cores were actually chosen from practical reasons related to availability of the components and winding issues. Still, in the following experiments standard vibration amplitudes do not lead to a case reaching the maximum energy capability.

The equivalent permeability of the chosen ferrite material is considerably lower than the permeability of some nanocrystalline materials 21, 22. The second core that is considered is thus made of Vitroperm 500F (VAC) which is a high permeability nanocrystalline material. The core is a $30 \times 20 \times 10$ toroid represented in Fig. 9 (a) and described in Table 2. In the end, this core offers a larger permeance $A_{L}$ when compared to the ferrite core. Note that the geometry of the toroid makes winding harder and the automation of the process would require a specific toroidal core winding machine.

\subsection{Practical design of the inductors}

The choice of the two magnetic cores is validated by the calculation of the criterion $c_{Q}$ for the two considered bending modes, from Eq. (26) and Tables 1 and 2. The filling factor $k_{u}$ is initially set to 0.5 in order to consider a full window area. The results are presented in Table 3 for the ferrite core and in Table 4 for the nanocrystalline toroid. Note that $c_{Q} \gg 1$, which means that both cores can satisfy the $L$ and $R$ specifications if the contribution of the parallel resistance presented in Eq. 20p is not too large. The 


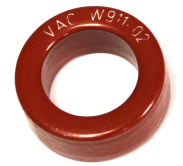

(a)
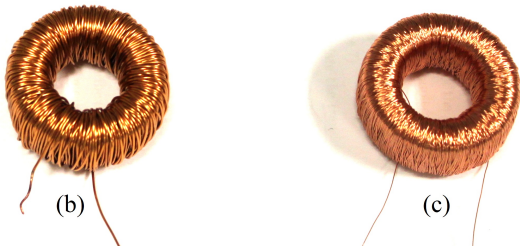

(c)

Figure 9: Nanocrystalline toroid: (a) Magnetic core before winding. (b) Wound with 296 turns $\left(d_{\mathrm{w}}=5 / 10 \mathrm{~mm}\right)$. (b) Wound with 1800 turns $\left(d_{\mathrm{w}}=2 / 10 \mathrm{~mm}\right)$.

Table 3: Inductor design characteristics with the ferrite core.

\begin{tabular}{|c||c|c|c|c|c|c|}
\cline { 2 - 7 } \multicolumn{1}{c|}{} & $\begin{array}{c}\boldsymbol{c}_{\boldsymbol{Q}} \\
(-)\end{array}$ & $\begin{array}{c}\boldsymbol{n} \\
(\text { turns })\end{array}$ & $\begin{array}{c}\boldsymbol{d}_{\mathbf{w}} \\
(\mathrm{mm})\end{array}$ & $\begin{array}{c}\boldsymbol{k}_{\boldsymbol{u}} \\
(-)\end{array}$ & $\begin{array}{c}\boldsymbol{I}_{\max }^{\boldsymbol{B}} \\
(\mathrm{mA})\end{array}$ & $\begin{array}{c}\boldsymbol{I}_{\max }^{\boldsymbol{J}} \\
(\mathrm{mA})\end{array}$ \\
\hline Mode 1 & 17 & 4520 & $5 / 100$ & 0.22 & 0.58 & 8.3 \\
\hline Mode 2 & 120 & 638 & $2 / 10$ & 0.49 & 4.1 & 130 \\
\hline
\end{tabular}

Table 4: Inductor design characteristics with the nanocrystalline toroid.

\begin{tabular}{|c||c|c|c|c|c|c|}
\cline { 2 - 7 } \multicolumn{1}{c|}{} & $\begin{array}{c}\boldsymbol{c}_{\boldsymbol{Q}} \\
(-)\end{array}$ & $\begin{array}{c}\boldsymbol{n} \\
(\text { turns })\end{array}$ & $\begin{array}{c}\boldsymbol{d}_{\mathrm{w}} \\
(\mathrm{mm})\end{array}$ & $\begin{array}{c}\boldsymbol{k}_{\boldsymbol{u}} \\
(-)\end{array}$ & $\begin{array}{c}\boldsymbol{I}_{\max }^{\boldsymbol{B}} \\
(\mathrm{mA})\end{array}$ & $\begin{array}{c}\boldsymbol{I}_{\max }^{\boldsymbol{J}} \\
(\mathrm{mA})\end{array}$ \\
\hline Mode 1 & 370 & 2416 & $2 / 10$ & 0.32 & 0.35 & 130 \\
\hline Mode 2 & 2500 & 341 & $5 / 10$ & 0.28 & 2.5 & 830 \\
\hline
\end{tabular}

number of turns is then computed from Eq. 22 and a standard wire diameter $d_{\mathrm{w}}=2 \sqrt{S_{\mathrm{w}} / \pi}$ is chosen by updating $k_{u}$ and ensuring that its value remains below 0.5. In the end, the maximum currents related to both conditions on the saturation flux density and the maximum current density are obtained from Eqs. (27) and $(28)$, where $J$ is set to $3 \mathrm{~A} / \mathrm{mm}^{2} 22$. For the present inductors involving cores with high permeance, the maximum current $I_{\max }^{B}$ is significantly lower than $I_{\max }^{J}$. In other words, an increase of the current would detune the resonant shunt before overheating the coil. This may be another advantage of the closed core inductors that would ensure the integrity of the electrical components.

The ferrite core reaches its limits when focusing on the first mode of the beam. Table 3 gives a solution with 4520 turns of 5/100 copper wire but its practical implementation is tough. Indeed, such a number of turns with a thin and thus delicate wire would require dedicated tools that were not available for the present application. This example shows that ferrite cores are sometimes not suitable when considering low frequency applications involving resonant shunts. Cores with larger $c_{Q}$ ratios are then necessary. Finally, the control of the first mode can still be satisfied with the nanocrystalline toroid. This core is wound by hand according to Table 4 for the first mode and for the second mode, which leads to the components in Figs. 9(b) and 9(c). The ferrite core is also wound for the control of the second modes but not for the first mode because of the aforementioned practical limit.

\subsection{Piezoelectric damping of vibrations}

The three inductors are successively connected to the pair of piezoelectric patches in order to reduce the vibration amplitude of the beam in Fig. 4(b). First, the damping of the second bending mode is targeted. The numbers of turns of the inductors are adjusted in order to get resonant shunts that are correctly tuned. The ferrite core is wound with 613 turns and the nanocrystalline toroid is wound with 296 turns. The difference with the theoretical values presented in Tables 3 and 4 is explained by the fact that the $A_{L}$ values appearing in Tables 2 are specified at $10 \mathrm{kHz}$. Yet, the permeance varies nonlinearly with respect to the frequency, as it has been observed for the inductance $\hat{L}(\omega)$ in Eq. 20. Over a frequency range below 


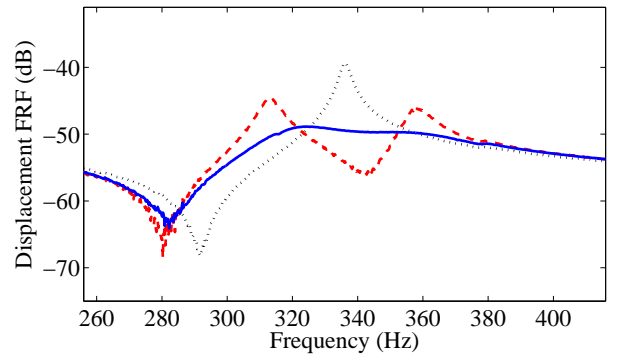

(a)

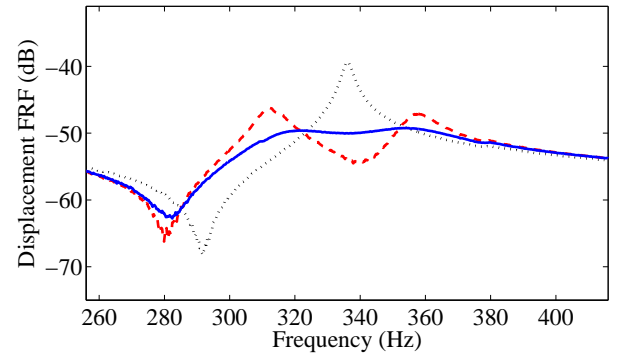

(b)

Figure 10: Damping of the second bending mode: (a) With the ferrite core $(n=613)$ : $(\cdots)$ open circuit, $(--)$ shunted on inductor without additional resistor, $(-)$ shunted on inductor with additional series resistor $R_{\mathrm{s}}^{+}=1.8 \mathrm{k} \Omega$. (b) With the nanocrystalline toroid $(n=296)$ : $(\cdots)$ open circuit, $(--)$ shunted on inductor without additional resistor, $(-)$ shunted on inductor with additional series resistor $R_{\mathrm{s}}^{+}=1.4 \mathrm{k} \Omega$.

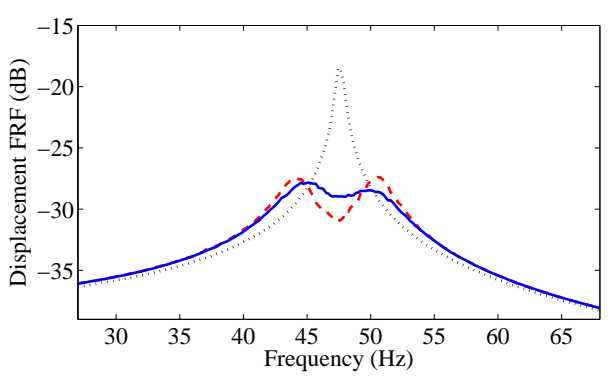

Figure 11: Damping of the first bending mode with the nanocrystalline toroid $(n=1800):(\cdots)$ open circuit, $(--)$ shunted on inductor without additional resistor, $(-)$ shunted on inductor with additional series resistor $R_{\mathrm{s}}^{+}=6.7 \mathrm{k} \Omega$.

$10 \mathrm{kHz}$, the $A_{L}$ values given by the core manufacturer are thus lower estimates that could be adjusted by measurement at the frequency of interest.

The closed core inductors offer an equivalent series resistance which is below the optimal shunt resistance. This is confirmed in Fig. 10, where it is seen that an external series resistance $R_{\mathrm{s}}^{+}$needs to be introduced in order to reach the optimal damping configuration. It is noticed that $R_{\mathrm{s}}^{+}$is below the optimal resistance $R=2.1 \mathrm{k} \Omega$. Yet, the series resistance of the wire is not significant as indicated by the large values of the $c_{Q}$ criterion for the chosen magnetic cores. This means that the magnetic losses modeled by the parallel resistance $R_{\mathrm{p}}$ cannot be neglected. Fortunately, its influence yields an equivalent series resistance that is lower than the optimal resistance.

The nanocrytalline toroid can satisfy the $327 \mathrm{H}$ requirement for the first mode of the beam. The toroid needs 1800 turns to reach this optimal inductance, which differs from the estimated value. Again, the main reason is that the equivalent permeance increases significantly when going to low frequency, especially with the considered nanocrystalline material. Figure 11 shows that the handmade inductor is able to provide a significant vibration reduction. Although some nonlinearities are observed 32, the magnetic flux density is actually far from the saturation limit. Indeed, the considered excitation amplitude causes an open-circuit displacement equal to $30 \mu \mathrm{m}$ at the end of the beam. This leads to a maximum current produced by the piezoelectric patches that is only about $14 \mu \mathrm{A}$, which is 25 times lower than the limit given in Table 4 .

\section{Conclusions}

A two-degree of freedom model gives optimal inductance and resistance values for a resonant shunt. This coupled model has been used to quantify the electrical current flowing through the inductor as well as the magnetic energy, which is required for an adequate design of the magnetic component. A criterion based on 
inductance and resistance specifications is proposed in order to choose a suitable magnetic core for resonant piezoelectric shunt applications. The experimental setup requires inductance up to $327 \mathrm{H}$ for a control with a resonant shunt. It is shown that such a high inductance value is not impractical for piezoelectric damping applications because of the low amplitudes of both the electrical current and the stored magnetic energy.

As custom designs are required to reach large inductance values with low series resistance, two cores made of soft magnetic materials are proposed: a RM core in ferrite and a nanocrystalline toroid. Ferrite cores offer decent permeabilities but some applications require nanocrystalline materials for even larger inductance. The inductor designs are successively validated by observing significant vibration reductions. Consequently, closed magnetic cores clearly extend the resonant shunt strategy to lower frequencies that were not reachable with standard passive inductors. Future perspectives include the optimization of the mass or dimensions of the magnetic cores. The interest of lower permeability materials or cores with air gap will be evaluated with respect to the potential increase in terms of energy density.

\section{Acknowledgments}

This work was funded by the French Ministry of National Education, Higher Education and Research through a three year scholarship for doctoral studies related to structural damping with piezoelectric devices.

\section{References}

[1] N. Hagood, A. von Flotow, Damping of structural vibrations with piezoelectric materials and passive electrical networks, Journal of Sound and Vibration 146 (1991) 243-268.

[2] J. P. Den Hartog, Mechanical Vibrations, McGraw-Hill, 1940.

[3] K. Liu, J. Liu, The damped dynamic vibration absorbers: revisited and new result, Journal of Sound and Vibration 284 (2005) 1181-1189.

[4] R. L. Forward, Electronic damping of vibrations in optical structures, Applied Optics 18 (1979) $690-697$.

[5] F. Bachmann, R. de Oliveira, A. Sigg, V. Schnyder, T. Delpero, R. Jaehne, A. E. Bergamini, V. Michaud, P. Ermanni, Passive damping of composite blades using embedded piezoelectric modules or shape memory alloy wires: a comparative study, Smart Materials and Structures 21 (2012) 075027.

[6] O. Thomas, J. Ducarne, J.-F. Deü, Performance of piezoelectric shunts for vibration reduction, Smart Materials and Structures $21(2012) 015008$.

[7] J.-S. Kim, U.-C. Jeong, J.-H. Seo, Y.-D. Kim, O.-D. Lee, J.-E. Oh, Structure borne noise control of a clamped panel using shunt damping system, Sensors and Actuators A: Physical 233 (2015) 330-348.

[8] B. Mokrani, R. Bastaits, M. Horodinca, I. Romanescu, I. Burda, R. Viguié, A. Preumont, Parallel piezoelectric shunt damping of rotationally periodic structures, Advances in Materials Science and Engineering 2015 (2015).

[9] J. J. Hollkamp, Multimodal passive vibration suppression with piezoelectric materials and resonant shunts, Journal of Intelligent Material Systems and Structures 5 (1994) 49-57.

[10] S.-Y. Wu, Method for multiple mode piezoelectric shunting with single PZT transducer for vibration control, Journal of Intelligent Material Systems and Structures 9 (1998) 991-998.

[11] A. J. Fleming, S. Behrens, S. O. R. Moheimani, Synthetic impedance for implementation of piezoelectric shunt-damping circuits, Electronics Letters 36 (2000) 1525-1526.

[12] A. J. Fleming, S. Behrens, S. O. R. Moheimani, Optimization and implementation of multimode piezoelectric shunt damping systems, IEEE/ASME Transactions on Mechatronics 7 (2002) 87-94.

[13] I. Giorgio, A. Culla, D. Vescovo, Multimode vibration control using several piezoelectric transducers shunted with a multiterminal network, Archive of Applied Mechanics 79 (2008) 859-879.

[14] S. Behrens, S. Moheimani, A. Fleming, Multiple mode current flowing passive piezoelectric shunt controller, Journal of Sound and Vibration 266 (2003) 929-942.

[15] L. Airoldi, M. Ruzzene, Wave propagation control in beams through periodic multi-branch shunts, Journal of Intelligent Material Systems and Structures 22 (2011) 1567-1579.

[16] A. J. Fleming, S. Behrens, S. O. R. Moheimani, Reducing the inductance requirements of piezoelectric shunt damping systems, Smart Materials and Structures 12 (2003) 57.

[17] G. Wang, S. Chen, J. Wen, Low-frequency locally resonant band gaps induced by arrays of resonant shunts with Antoniou's circuit: experimental investigation on beams, Smart Materials and Structures 20 (2011) 015026.

[18] L. Airoldi, M. Ruzzene, Design of tunable acoustic metamaterials through periodic arrays of resonant shunted piezos, New Journal of Physics 13 (2011) 113010

[19] F. Casadei, M. Ruzzene, L. Dozio, K. A. Cunefare, Broadband vibration control through periodic arrays of resonant shunts: experimental investigation on plates, Smart Materials and Structures 19 (2010) 015002.

[20] S. Chen, G. Wang, J. Wen, X. Wen, Wave propagation and attenuation in plates with periodic arrays of shunted piezopatches, Journal of Sound and Vibration 332 (2013) 1520-1532.

[21] C. W. T. McLyman, Transformer and Inductor Design Handbook, CRC Press, 2004. 
[22] B. Multon, Composants passifs de l'électronique de puissance (magnétiques et capacitifs), Lecture, 2015. URL: https: //cel.archives-ouvertes.fr/cel-01246811

[23] Epcos, Ferrites and accessories. General - Definitions, 2006.

[24] B. Lossouarn, J.-F. Deü, M. Aucejo, Multimodal vibration damping of a beam with a periodic array of piezoelectric patches connected to a passive electrical network, Smart Materials and Structures 24 (2015) 115037.

[25] B. Lossouarn, J.-F. Deü, M. Aucejo, K. A. Cunefare, Multimodal vibration damping of a plate by piezoelectric coupling to its analogous electrical network, Smart Materials and Structures 25 (2016) 115042.

[26] A. Bloch, Electromechanical analogies and their use for the analysis of mechanical and electromechanical systems, Journal of the Institution of Electrical Engineers - Part I: General 92 (1945) 157-169.

[27] Y. S. Cho, Y. Pak, C. S. Han, S. K. Ha, Five-port equivalent electric circuit of piezoelectric bimorph beam, Sensors and Actuators A: Physical 84 (2000) 140-148.

[28] R. P. Paganelli, A. Romani, A. Golfarelli, M. Magi, E. Sangiorgi, M. Tartagni, Modeling and characterization of piezoelectric transducers by means of scattering parameters. part i: Theory, Sensors and Actuators A: Physical 160 (2010) 9-18.

[29] P. Soltani, G. Kerschen, G. Tondreau, A. Deraemaeker, Piezoelectric vibration damping using resonant shunt circuits: an exact solution, Smart Materials and Structures 23 (2014) 125014.

[30] J. Ducarne, O. Thomas, J.-F. Deü, Placement and dimension optimization of shunted piezoelectric patches for vibration reduction, Journal of Sound and Vibration 331 (2012) 3286-3303.

[31] C. Maurini, J. Pouget, F. dell'Isola, Extension of the Euler-Bernoulli model of piezoelectric laminates to include 3D effects via a mixed approach, Computers \& Structures 84 (2006) 1438 - 1458. Composite Adaptive Structures: Modelling and Simulation.

[32] B. Lossouarn, O. Thierry, M. Aucejo, J.-F. Deü, Comparison of passive inductor designs for piezoelectric shunt damping, 2016. doi $10.1117 / 12.2219200$ 